\title{
CONSIDERACIONES SOBRE LA VALORACIÓN TECNOLÓGICA EN LA BASE DE LA PIRÁMIDE*
}

\author{
CLAUDIA NELCY JIMÉNEZ $Z^{* * *} \&$ OSCAR CASTELLANOS ${ }^{* * *}$ \\ UNIVERSIDAD NACIONAL DE COLOMBIA
}

Recibido/ Received/ Recebido: 06/12/2013 - Aceptado/ Accepted/Aprovado: 29/05/2014

\begin{abstract}
Resumen
El concepto de "Base de la Pirámide" se refiere a gran parte de la población mundial de más escasos recursos y a las opciones para atender sus necesidades. Este artículo de reflexión tiene el objetivo de analizar un aspecto de la gestión tecnológica en la base de la pirámide: la valoración tecnológica orientada a los productores locales, en su mayoría pequeñas y medianas empresas - Pymes. Tomando en cuenta las características de las tecnologías que prevalecen en estas empresas, así como las escasas capacidades de innovación, como resultado de este artículo se plantean algunas consideraciones sobre la denominada valoración tecnológica ampliada, que no se limita a determinar el valor económico de un activo tecnológico, sino que toma en cuenta aspectos intangibles como las capacidades tecnológicas, los procesos de aprendizaje y la acumulación tecnológica que pueden seguir dichas empresas para lograr innovaciones y mayor desarrollo en tecnología, en aras de disminuir las brechas frente a los países industrializados.
\end{abstract}

Palabras clave: Base de la pirámide, Innovación tecnológica, Valoración tecnológica, Evaluación tecnológica, Pymes, Latinoamérica.

\section{CONSIDERATIONS ON TECHNOLOGICAL ASSESSMENT AT THE PYRAMID BASE}

\begin{abstract}
The concept of "pyramid base" refers to most of world's population with limited resources and options to respond to their needs. This reflective article aims to analyze the technological management at the pyramid base: technological assessment targeted to local producers, most of them small and medium enterprises-SMEs. Considering technology characteristics often used in these enterprises, as well as, the limited innovation capacity. We propose some considerations on the so-called extended technological assessment. We consider that this process is not limited to determining economic value of a technological asset, but takes into account intangibles such as technology skills, learning and technological accumulation that these companies can continue to achieve for greater innovation and technological development in order to reduce their gaps compared to industrialized countries.
\end{abstract}

Artículo de reflexión derivado de la tesis doctoral titulada "Propuesta para la valoración tecnológica desde la perspectiva de la Síntesis Evolutiva Moderna".

** Ingeniera Química, Magíster en Administración, Candidata a Ph.D. en Ingeniería. Profesora Facultad de Minas, Universidad Nacional de Colombia, Sede Medellín. Investigadora Grupo Biogestión. Correo electrónico: cnjimenezh@unal.edu.co. Tel: (57-4) 4255200. Dirección postal: Calle 44 No 45-67, Unidad Camilo Torres Bloque B5 Of. 303, Universidad Nacional de Colombia, Bogotá, Colombia.

**** Ingeniero Químico, Magíster en Administración, Magíster en Ciencias Técnicas, Ph.D. en Ciencias. Profesor Facultad de Ingeniería, Universidad Nacional de Colombia, Sede Bogotá. Director del Grupo de Investigación y Desarrollo en Gestión, Productividad y Competitividad Biogestión. Correo electrónico: ofcastellanosd@unal.edu.co. Tel: (57-1) 3165000 Ext. 10730. Dirección postal: Calle 44 No 45-67, Unidad Camilo Torres Bloque B5 Of. 303, Universidad Nacional de Colombia, Bogotá, Colombia. 
Keywords: Pyramid base, Technological innovation, Technological assessment, Technological evaluation, SMEs, Latin-American.

\title{
CONSIDERAÇÕES SOBRE A VALORIZAÇÃO TECNOLÓGICA NA BASE DA PIRÁMIDE
}

\begin{abstract}
Resumo
O conceito de "base da pirâmide" refere-se à grande parte da população mundial com escassos recursos e opções para atender suas necessidades. Este artigo de reflexão tem o objetivo de analisar um aspecto da gestão tecnológica na base da pirâmide: a valorização tecnológica orientada aos produtores locais, em sua maioria pequenas e médias empresas - Pymes. Considerando as características das tecnologias que prevalecem nestas empresas, bem como as escassas capacidades de inovação, propusemos algumas considerações sobre a denominada valorização tecnológica ampliada. Consideramos que este processo não se limita a determinar o valor econômico de um ativo tecnológico, mas considera aspectos intangíveis como as capacidades tecnológicas, os processos de aprendizagem e acumulação tecnológica que podem seguir referidas empresas para conseguir inovações e maior desenvolvimento tecnológico, com o fim de diminuir as brechas frente aos países industrializados.

Palavras chave: Base da pirâmide, Inovação tecnológica, Valorização tecnológica, Avaliação tecnológica, Pymes, América Latina.

Jiménez, C. \& Castellanos, O. (2014) Consideraciones sobre la valoración tecnológica en la base de la pirámide. En: Revista de la Facultad de Ciencias Económicas de la Universidad Militar Nueva Granada. rev.fac.cienc.econ, XXII (2).
\end{abstract}

JEL: O31, O32, O39.

\section{Introducción}

El concepto de Base de la pirámide hace referencia a un segmento sociodemográfico en el estrato inferior de renta de la población mundial, compuesto por cuatro mil millones de personas - cuyos ingresos diarios en dólares corrientes son inferiores a 3,35 en Brasil, 2,11 en China, 1,89 en Ghana y 1,56 en India - correspondientes al $72 \%$ de la población mundial, que en continentes como Asia, África o regiones como Europa del Este, América Latina y el Caribe, representa a la abrumadora mayoría; se estima que la base de la pirámide constituye un mercado de consumo de 1,3 billones de dólares destinado principalmente a los sectores alimentos, energía, vivienda, transporte, salud, tecnologías de información y comunicación -TIC, y agua (Casado, Lariu, Mutis \& Sánchez, 2009).
La atención de las necesidades de la población de esta zona requiere de la fabricación de productos que estén a su alcance; sin embargo, los productores locales deben emplear los recursos tecnológicos disponibles, que tienen características particulares, y de los cuales resulta clave conocer el valor. En los procesos de valoración en la base de la pirámide, las capacidades tecnológicas constituyen un elemento fundamental (Jiménez, Cristancho \& Castellanos, 2011).

Este artículo se enfoca en realizar una reflexión en torno a la valoración tecnológica en el contexto particular de la base de la pirámide, partiendo de algunos aspectos relevantes al estudiar esta función de la gestión tecnológica:

- Los métodos y modelos de valoración tecnológica han sido cuestionados en diferentes épocas, 
por considerarse insuficientes en la inclusión de aspectos clave como el conocimiento incorporado en los activos tecnológicos, su complejidad y el entorno en el que dichos activos se encuentran (Hunt, Probert, Wong \& Phaal, 2003; Eichner, Gemünden \& Kautzsch, 2007).

- Kaplan (1986), Kakati \& Dhar (1991) y Raafat (2002) han sugerido involucrar factores estratégicos, como los cualitativos, de manera más explícita en los procesos de valoración tecnológica, $e$ incluso se ha recomendado el uso de aproximaciones mixtas o híbridas en las que se consideren tanto factores cuantitativos como cualitativos. Small \& Chen (1997, citados en Doerr, Gates \& Mutty, 2006) empíricamente evidenciaron que muchas empresas no usan técnicas de evaluación sofisticada, pero aquellas que emplean una aproximación amplia - incluyendo aspectos cuantitativos y cualitativos - tienden a lograr mejores resultados en la implementación de la tecnología valorada.

Las diferentes críticas que ha recibido la valoración tecnológica evidencian que ha estado influenciada por la visión mecanicista y los esquemas conceptuales tradicionales de la ingeniería industrial, procedentes de los preceptos del Taylorismo y el Fayolismo, de manera que esta valoración se fundamenta en epistemologías efectivas pero insuficientes (Jiménez \& Castellanos, 2011, 2013). Partiendo de estas evidencias epistemológicas, en este documento se analiza la valoración tecnológica y la necesidad de una visión más amplia, mediante una reflexión a la luz de las características particulares del contexto de la base de la pirámide.

Metodológicamente, el artículo se basa en el análisis de literatura relevante sobre los temas principales: las características, las tecnologías y las capacidades tecnológicas en la base de la pirámide, junto con el tema de valoración tecnológica. Inicialmente se aborda el concepto de Base de la pirámide y su contextualización al caso latinoamericano; luego se analiza el papel de la tecnología en la fabricación de bienes para este segmento de la pirámide socioeconómica, y posteriormente se revisa el tema de capacidades tecnológicas y su incidencia en contextos de menor desarrollo. Por último, se formulan varias consideraciones sobre los procesos de valoración tecnológica en el ámbito de las empresas que atienden a la base de la pirámide, las cuales retoman una conceptualización amplia de la tecnología que incluye las capacidades tecnológicas.

\section{La base de la pirámide y las economías en desarrollo: el caso latinoamericano}

Prahalad \& Hart (2002) plantearon los fundamentos del concepto de Base de la Pirámide, señalando que más allá de los pocos ricos y los consumidores de ingresos medios en los países en desarrollo, quienes deben ser el foco de las estrategias de mercado de las corporaciones multinacionales son los miles de millones de "aspirantes pobres" que se incorporan a la economía de mercado por primera vez.

Prahalad (2005) plantea que existe una opción para enfrentar la pobreza, diferente a la visión asistencialista que ha primado en el tema; señala que puede hablarse de una pirámide socioeconómica (Gráfica 1) en cuya parte más alta se encuentra la minoría de la población mundial que dispone de una gran cantidad de recursos, mientras que en la base se ubican cuatro mil millones de pobres, para quienes ninguna campaña gubernamental, de las instituciones multilaterales o de las agencias internacionales ha proporcionado soluciones efectivas.

Por tanto, debe intentarse un enfoque diferente que implica trabajar conjuntamente con los pobres, buscando posibilidades en las cuales se les involucre activamente, generando beneficios tanto para ellos como para las empresas. Para tal fin son necesarios la innovación, el acceso y la disponibilidad de productos y servicios orientados a la base de la pirámide.

Si bien el concepto se dirigía en un comienzo a las grandes multinacionales, se ha reconocido que las pequeñas y medianas empresas locales están mejor preparadas para atender el mercado de la base de la pirámide, ya que conocen muy bien a las comunidades en las cuales están insertas y generan empleo allí mismo (BID, 2011). Por ello, el protocolo 2.0 de la base de la pirámide (BoP 2.0) involucra el con- 
Gráfica 1. La pirámide económica ${ }^{1}$

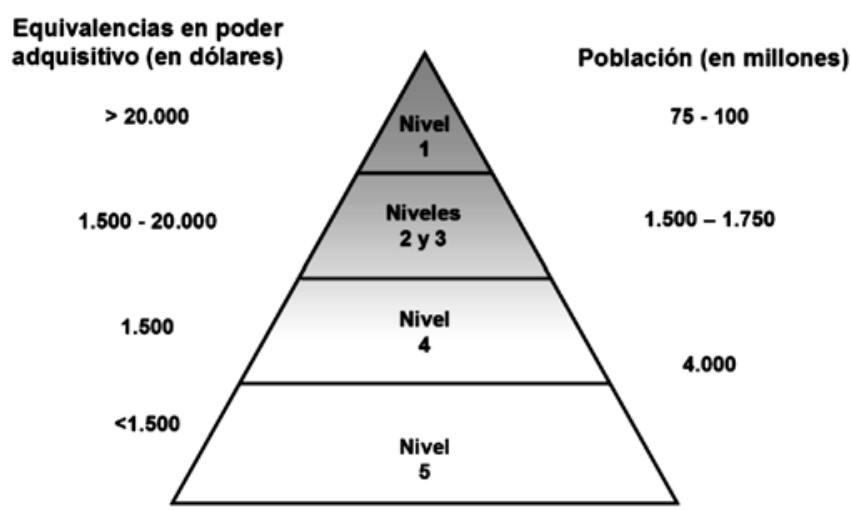

cepto de co-creación de valor, dejando de lado la visión del pobre como consumidor, para comenzar a verlo como socio de negocios, con el cual tanto las grandes empresas multinacionales como las Pymes locales pueden integrar capacidades y generar compromisos compartidos hacia una nueva propuesta de valor (Simanis et al., 2008, citado en Calderón \& Silva, 2009). En este sentido, Kolk, Rivera-Santos \& Rufín (2013) muestran como la literatura que aborda las iniciativas sobre el concepto de Base de la Pirámide, reporta la participación cada vez mayor de los pobres en toda la cadena de valor (Gráfica 2).
Gran parte de la población de América Latina se encuentra en la base de la pirámide, en donde, según Salom \& Shulterbrandt (2002), se requiere estudiar y comprender la situación caracterizada por problemáticas como el desequilibrio económico, los desórdenes sociales, la pobreza generalizada y la corrupción social, empresarial y gubernamental, los cuales están directamente relacionados y afectan los aparatos productivos nacionales; la escasez de estadísticas en muchas áreas se añade a las dificultades para encontrar soluciones definitivas a estos problemas.

Gráfica 2. Posición del pobre en la cadena de valor en las iniciativas de base de la pirámide²

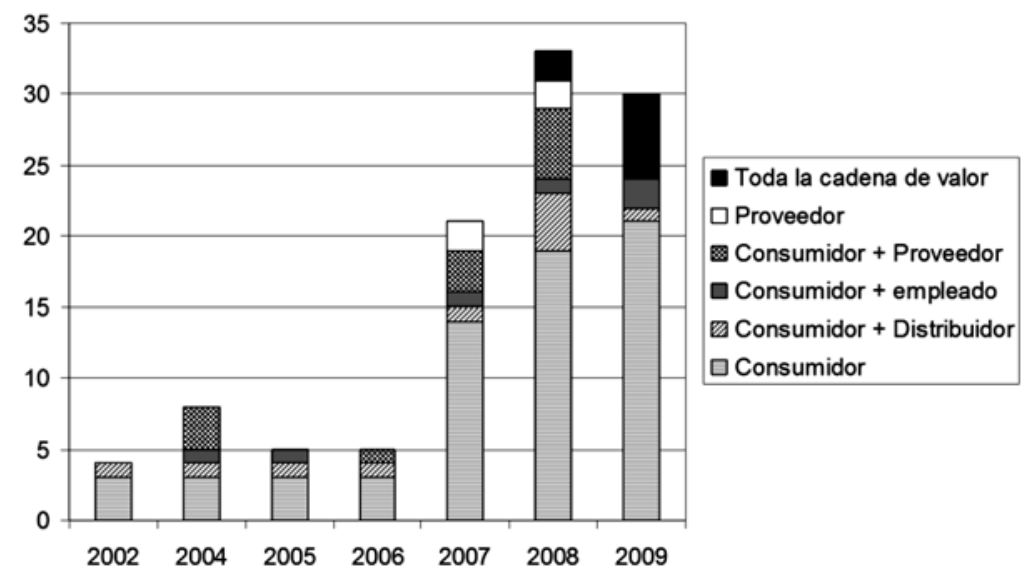

Fuente: Prahalad (2005).

Fuente: Kolk et al. (2013). 
Latinoamérica ofrece un escenario particular para el desarrollo productivo y tecnológico, y aunque en vías de mejora, aún presenta condiciones que dificultan estos procesos. Al hablar de economías de menor desarrollo, en el caso de Latinoamérica existen características comunes en cuanto al progreso tecnológico (Malaver \& Vargas, 2006): principalmente exógeno, inmediatista, producto de procesos informales de innovación de carácter incremental. El 99\% de las empresas de la región es de tamaño pequeño o mediano, con un elevado aporte en cuanto a generación de empleo mientras que su contribución a la producción es baja, lo cual es reflejo de la estructura productiva heterogénea, la especialización en productos de bajo valor agregado y su reducida participación en las exportaciones, inferior a $5 \%$ en la mayoría de los países (OCDE \& CEPAL, 2012).

Las Pymes latinoamericanas se concentran en los sectores comercio, servicios informales y manufacturas básicas, que demandan poco conocimiento, pero su comportamiento innovador es difícil de estudiar, ya que generalmente se hace a través de encuestas de innovación no comparables entre países. Algunas regularidades identificadas son el predominio de innovaciones de proceso y de actividades de I+D poco significativas. Entre un 20 y un $25 \%$ de las Pymes han tenido proyectos de innovación. Se destaca la informalidad de las estrategias de innovación, aunque las empresas de sectores intensivos en conocimiento muestran mayor formalidad, y un nivel de inversión en $\mathrm{I}+\mathrm{D}$ más elevado y estable en el tiempo. Sin embargo, su baja interacción con otras organizaciones disminuye el impacto de estas estrategias (CEPAL, 2011; OCDE \& CEPAL, 2012).

\section{El papel de la tecnología en la base de la pirámide}

Prahalad (2010) analizó once casos exitosos en la base de la pirámide en países como India y Brasil. Tomándolos como referencia, en la Tabla 1 se identifican los productos y servicios que se pusieron a disposición de la población en la base de la pirámide, caracterizando sus procesos de fabricación o generación como tradicionales o innovadores.
En los casos de la Tabla 1 se aprecia que para ofrecer productos y servicios en la base de la pirámide es necesario ser innovadores. Sin embargo, también es posible ver que la fabricación de productos generalmente se hace de manera tradicional, con las tecnologías comúnmente disponibles en países de menor desarrollo, mientras que las innovaciones ocurren en la logística y el mercadeo, para garantizar que estos productos lleguen a los consumidores (Jiménez \& Castellanos, 2012). Lo mismo ocurre con los casos que se han analizado en escenarios como la primera y segunda versión del Foro Base Internacional para el desarrollo de los mercados en la base de la pirámide en Latinoamérica y el Caribe, organizado por el Banco Interamericano de Desarrollo, realizado en 2011 en Brasil y en 2013 en Colombia, en donde sobresalen los mecanismos de acercamiento al microcrédito y a productos de consumo masivo como las bebidas gaseosas o los derivados lácteos.

Al respecto, Prahalad (2010) señala que existe la suposición de que en la base de la pirámide los mercados no son de alta tecnología, pero muchas empresas han tenido que recurrir a las tecnologías más modernas para lograr calidad y oportunidad en la entrega, especialmente Tecnologías de Información y Comunicación - TICs; no obstante, no necesariamente se emplean en los procesos de fabricación, sino que inciden en otros aspectos como la comercialización y la entrega. Por ello, el propio Prahalad menciona que las tecnologías avanzadas deben combinarse con las infraestructuras existentes (deficientes o regulares) para generar soluciones pertinentes a los mercados de la base de la pirámide.

\subsection{Tecnologías en la base de la pirámide}

La transferencia y la apropiación internacional de tecnologías han sido clave en la interpretación del desarrollo en contextos poco industrializados y en la formulación de políticas e instrumentos específicos (Vaccarezza, 2011), ya que la generación de tecnologías propias aún no tiene un papel significativo. No obstante, se ha afirmado que el desarrollo tecnológico endógeno es la mejor vía que tienen los países rezagados, como aquellos de la base de la pirámide, para aprovechar los recursos locales y generar valor agregado a partir de ellos (Jaramillo, Lugones \& Sa- 
Tabla 1. Casos de éxito en la base de la pirámide

\begin{tabular}{|c|c|c|c|}
\hline Caso & País & Producto o servicio & $\begin{array}{l}\text { Proceso de fabricación o generación } \\
\text { (tradicional - innovación) }\end{array}$ \\
\hline Jaipur Rugs & India & Alfombras & $\begin{array}{l}\text { Tradicional (telar). Se innovó en la forma de vinculación de } \\
\text { los tejedores y en la organización del negocio para inter- } \\
\text { nacionalizarlo. }\end{array}$ \\
\hline Casas Bahía & Brasil & $\begin{array}{l}\text { Ventas mediante tiendas minoristas (electrodo- } \\
\text { mésticos, muebles, etc.) }\end{array}$ & $\begin{array}{l}\text { Innovación en la financiación para la compra de sus mer- } \\
\text { cancías. }\end{array}$ \\
\hline Cemex & México & Financiación de materiales de construcción & $\begin{array}{l}\text { Innovación en el mecanismo de financiación mediante or- } \\
\text { ganización de la comunidad. }\end{array}$ \\
\hline Jabón Lifebuoy & India & Jabón para manos & $\begin{array}{l}\text { Producción tradicional - Innovación en el mercadeo para } \\
\text { cambiar la cultura de la falta de uso de jabón en el lavado } \\
\text { de manos. }\end{array}$ \\
\hline Sal yodada & India & Sal para consumo humano & $\begin{array}{l}\text { La obtención de sal es tradicional. Se innovó en la forma } \\
\text { de inclusión del yodo en la sal para evitar su pérdida antes } \\
\text { del consumo. }\end{array}$ \\
\hline Jaipur foot & India & Prótesis para discapacitados & $\begin{array}{l}\text { Innovación para lograr productos baratos y de calidad, con } \\
\text { cortos tiempos de adaptación de los pacientes a la prótesis. }\end{array}$ \\
\hline Aravind Eye Care & India & Servicios de oftalmología y cirugía & $\begin{array}{l}\text { Innovación en la forma de prestar el servicio, con campa- } \\
\text { mentos y jornadas de atención. }\end{array}$ \\
\hline $\begin{array}{l}\text { ICICl: Servicios financieros } \\
\text { para los pobres }\end{array}$ & India & Servicios financieros & $\begin{array}{l}\text { Innovación en la forma de prestar el servicio de microcrédi- } \\
\text { to para quienes no poseen respaldo financiero. }\end{array}$ \\
\hline ITC e-Choupal & India & $\begin{array}{l}\text { Servicios de comercialización de productos agrí- } \\
\text { colas }\end{array}$ & $\begin{array}{l}\text { Innovación por la introducción de TICs en la comercializa- } \\
\text { ción de productos agrícolas. }\end{array}$ \\
\hline Voxiva & Perú & $\begin{array}{l}\text { Servicios de información temprana sobre enfer- } \\
\text { medades y epidemias }\end{array}$ & $\begin{array}{l}\text { Innovación en la plataforma de difusión de la información, } \\
\text { empleando teléfonos móviles. }\end{array}$ \\
\hline $\mathrm{E}+$ Co. Energía para todos & Nicaragua & $\begin{array}{l}\text { Generación y suministro de energía eléctrica a } \\
\text { partir de fuentes alternas }\end{array}$ & $\begin{array}{l}\text { Innovación en la promoción de empresas locales generado- } \\
\text { ras de energía con fuentes alternas. }\end{array}$ \\
\hline
\end{tabular}

lazar, 2001). Sin embargo, sigue siendo un reto que llegue a comprenderse que la ciencia y la tecnología atañen a toda la sociedad, y no solo a científicos y tecnólogos (Albornoz, 2001). Sin desconocer este interesante debate sobre las causas y consecuencias del rezago tecnológico en regiones como Latinoamérica, en este artículo se acudirá al concepto de ciclo de vida de la tecnología y al de oleadas tecnológicas, con el fin de identificar las características principales de las tecnologías empleadas por los productores en la base de la pirámide.

El ciclo de vida de una tecnología abarca las fases de investigación y desarrollo, crecimiento, expansión, madurez y declive. Cada una de estas etapas implica inversión de recursos y a medida que se avanza en ellas, el aporte potencial de la tecnología a la competitividad es mayor, si bien las tecnologías en declive pierden importancia y se hace necesario sustituirlas. Este ciclo que cumplen tecnologías particulares se asemeja a lo que Pérez $(2005,2009)$ ha denominado revolución tecnológica u oleada de transformación en el contexto global (Gráfica 3), cuyo primer periodo se caracteriza por la instalación del nuevo paradigma con el surgimiento de innovaciones radicales sobre las que se articulan las innovaciones subsiguientes; este periodo abarca dos fases, en donde inicialmente se da un rápido crecimiento e innovación, y en la segunda fase ocurre una difusión amplia del paradigma, con el surgimiento de sistemas tecnológicos y ampliación de mercados. Posteriormente llega un periodo de despliegue de todo el potencial

3 Fuente: adaptado de Prahalad (2010). 
Gráfica 3. Ciclo de vida de la tecnología y de las revoluciones tecnológicas ${ }^{4}$

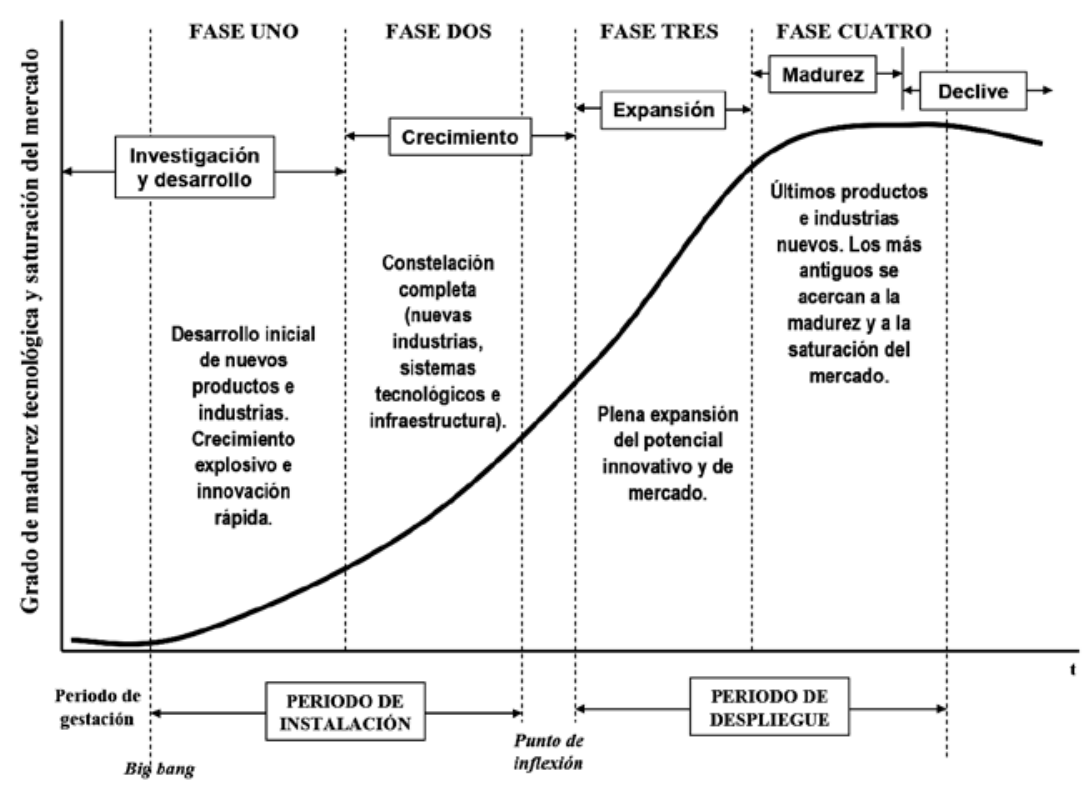

del nuevo paradigma, en donde, en una tercera fase, este se expande totalmente, y en la cuarta fase llega la madurez, de manera que el potencial del paradigma comienza a encontrar límites, y se presentan retornos decrecientes de la inversión en innovación tecnológica.

Para contar con tecnologías en las primeras fases del ciclo tecnológico se requiere de altas capacidades, disponibles principalmente en organizaciones como las grandes corporaciones multinacionales y empresas de países industrializados, que pueden invertir altas sumas en I+D para generar sus propias tecnologías y asumir el elevado riesgo que ello implica. Así mismo, de acuerdo con Fúquene (2011), en la fase de crecimiento del ciclo tecnológico, los nuevos desarrollos comienzan a divulgarse, por lo que se requiere alta inversión para su instalación y asimilación, así como elevadas capacidades tecnológicas, lo cual no es muy común en países en desarrollo.

De igual forma, el surgimiento de un nuevo paradigma en una revolución tecnológica depende en gran medida de las organizaciones capaces de generar innovaciones radicales en el momento en que la obsolescencia del paradigma prevaleciente comienza a evidenciarse. Son los países desarrollados los que se incorporan más rápidamente a esta nueva oleada tecnológica, y según Pérez (2005), plantean una tensión respecto a las perspectivas de los países rezagados. De esta manera, el periodo de instalación de la revolución tecnológica es un espacio para que organizaciones y países líderes tecnológica y económicamente se destaquen, mientras que los seguidores pueden incorporarse a esta revolución en el periodo de despliegue. Algo similar puede decirse en cuanto a las etapas del ciclo de vida tecnológico: principalmente los países y organizaciones más avanzados pueden cumplir con todo el ciclo, mientas que empresas y países en desarrollo deben optar por tecnologías en las etapas de expansión y madurez, e incluso acomodarse al uso de tecnologías en declive.

De acuerdo con Pérez (2001) se ha evidenciado que se requiere de un uso más intensivo de mano de obra y de personal altamente calificado durante las

4 Fuente: adaptado de Pérez (2005) y The Economist (2008). 
fases iniciales de la tecnología. En las etapas finales del ciclo de vida, la tecnología se utiliza en procesos altamente estandarizados, mecanizados y automatizados. De acuerdo con las dinámicas de las oleadas tecnológicas, las tecnologías maduras encuentran fuerzas que las expulsan hacia países con recursos financieros escasos, donde fuerzas complementarias pueden atraerlas para sus procesos de desarrollo.

Por tanto, puede afirmarse que en la base de la pirámide predominan tecnologías en las últimas etapas del ciclo tecnológico, y de manera habitual pueden encontrarse en sectores industriales tradicionales como el agroindustrial o el textil. Estos recursos tecnológicos disponibles localmente para la producción de bienes y servicios orientados a la base de la pirámide, deben articularse con tecnologías emergentes para lograr la atención oportuna y pertinente de las necesidades de la población de esta zona, lo que de acuerdo con Prahalad (2010), requiere de innovación gerencial que permita repensar la forma, la funcionalidad, los canales y los costos de distribución de los productos orientados a los más pobres.

Pérez (2001) señala que las tecnologías maduras tienen un potencial mínimo para producir beneficios y mejorar la productividad, además de que enfrentan mercados estancados, por lo cual la fase de madurez como punto de partida es costosa, poco rentable y poco prometedora. "Con todo, se trata probablemente del mejor punto de partida para crear una plataforma básica de industrialización, generar capacidad de aprendizaje y establecer la infraestructura básica y otros factores externos requeridos para respaldar un esfuerzo de desarrollo" (Pérez, 2001, 118).

A partir de lo expuesto, puede señalarse que la innovación para producir en la base de la pirámide se asocia con el empleo de tecnologías en las últimas fases del ciclo de vida, pero principalmente con la forma en que se aprovechan dichas tecnologías, es decir, el énfasis está en la capacidad de gestionar eficientemente estos recursos y en generar aprendizaje en torno a ellos, para lograr mejoras en procesos y productos que puedan considerarse como innovaciones. En la siguiente sección se profundiza en este tema.

\section{Las capacidades tecnológicas en países en desarrollo}

Habitualmente la capacidad de generar tecnología se asocia casi exclusivamente con los sistemas físicos utilizados en la producción de bienes terminados con alto valor agregado. Para ampliar esta perspectiva, Leonard-Barton (1998) identifica que la competencia de los negocios se logra mediante el desarrollo de habilidades tecnológicas propias, por lo cual la capacidad tecnológica de la empresa depende del desarrollo simultáneo en sistemas físicos (equipos e instalaciones), sistemas de gestión, habilidades y conocimientos de sus empleados, normas y valores de la empresa.

El avance tecnológico logrado por una organización está ligado directamente a las posibilidades de su entorno; por ello, la investigación sobre capacidades tecnológicas debe enfocarse en dos dimensiones: el desempeño de los sistemas productivos para países desarrollados y para economías en desarrollo. Las perspectivas tienden a ser diferenciadas puesto que en una economía desarrollada, como la mayoría de empresas están en la frontera tecnológica, ya existen tales capacidades, y por tanto, la investigación se centra en la adecuada identificación de aquellas que generen ventajas en el mercado, la consolidación de los mecanismos de apoyo a su promoción, la mejor comprensión de sus componentes, la rutinización de actividades y la óptima renovación de las mismas. Entre tanto, en países en desarrollo es habitual que los sistemas productivos no dispongan de un cúmulo de capacidades tecnológicas, por lo que el debate surge en torno a las actividades requeridas para su construcción y acumulación (Figueiredo, 2001a).

Bell \& Pavitt (1993) señalan que tradicionalmente se ha entendido que únicamente a través de la difusión -pasiva- de tecnología, los países en desarrollo pueden lograr el crecimiento industrial, desconociendo el valor e importancia de los recursos intangibles requeridos para generar y manejar el cambio técnico, al interpretarlos solo como complemento menor de la capacidad de producción. Se ha tenido un concepto errado de difusión, alejado del de innovación, que en realidad implica más que la adquisición de maquinaria y la asimilación relacionada con el knowhow de producción, considerando que los países que 
adoptan y usan tecnología pueden desempeñar un papel creativo al moldear las innovaciones adquiridas, ajustarlas a condiciones particulares y mejorarlas para lograr desempeños mayores.

Las economías en desarrollo tienen diferentes mecanismos para adquirir capacidades tecnológicas, como los mencionados por Bell \& Albu (1999):

- A través de actividades tecnológicas internas (observación de rutinas, mantenimiento de máquinas, experimentación, ingeniería inversa).

- De fuentes externas, como subproducto de varias clases de interacción.

- Por la formación de capital humano al interior de las firmas.

Así mismo, como lo indica Viotti (2002), las empresas son los actores principales, por lo menos en potencia, del proceso de cambio técnico y crecimiento de los países, incluso si son consideradas como no innovadoras en el contexto de economías en desarrollo. Por ello, el estudio de las capacidades tecnológicas de las empresas de países en desarrollo y de sus procesos de aprendizaje y acumulación tecnológica, pueden contribuir a un mejor entendimiento de su realidad para promover líneas de acción que lleven a un mayor crecimiento tecnológico, económico y social del país en el cual funcionan.

Figueiredo (2001b) establece que el desempeño tecnológico de las organizaciones se puede describir con la construcción de dos marcos de análisis: la acumulación de capacidades y los procesos de aprendizaje tecnológico; estos modelos se contextualizan respecto al desarrollo económico de los países y su formación tecnológica. El primer modelo se relaciona con las formas y caminos por donde se desarrolla la acumulación de capacidades tecnológicas, así como la trayectoria, dirección y la tasa de acumulación. El segundo modelo se refiere a los diversos procesos de adquisición de conocimientos por las personas y los procesos a través de los cuales el aprendizaje individual se convierte en el aprendizaje organizacional.

Existe una articulación directa entre los dos modelos de análisis ya que el aprendizaje tecnológico se refiere a los diversos procesos que permiten a la empre- sa acumular capacidades tecnológicas en el tiempo (Jaramillo et al., 2001); estos procesos son internos, acumulativos y colectivos e implican la adquisición de capacidades tanto por los individuos como por las organizaciones (Maculan, Jiménez \& Castellanos, 2014). El aprendizaje permite que los conocimientos técnicos de las personas, que por naturaleza son tácitos, se transformen en sistemas físicos, procesos, procedimientos, prácticas y productos y servicios de la organización (Figueiredo, 2001b, 2004).

Asociado con los esfuerzos internos de las empresas para adaptar y perfeccionar la tecnología por ellas adquirida, está el concepto de la capacidad tecnológica, entendida como el conjunto de rutinas apropiadas exclusivamente por la organización, que le permiten diferenciarse en el manejo del recurso tecnológico (Dutrénit, 2001). Bell \& Pavitt (1993) definen las capacidades tecnológicas como los recursos necesarios para generar y gestionar el cambio tecnológico, incluyendo las habilidades, conocimientos y experiencia, las estructuras institucionales y las redes de conexiones, y las diferencian de la capacidad industrial de producción, que está representada en los recursos (habilidades, conocimientos y bienes de capital) requeridos para producir bienes con la tecnología disponible.

Viotti (2002) habla de las capacidades tecnológicas básicas, de las cuales depende el desarrollo de los países, como las habilidades de las firmas para crear ingresos, incrementar la productividad, competir y crecer. Referencia tres categorías básicas:

- Capacidad de producción: conocimiento, habilidades y otras condiciones requeridas para el proceso de producción.

- Capacidad de mejoramiento: conocimiento, habilidades y otras condiciones requeridas para la actualización continua e incremental del diseño y características de desempeño de los productos, y de la tecnología de proceso.

- Capacidad de innovación: conocimiento, habilidades y otras condiciones requeridas para la creación de nuevas tecnologías (diseño y características centrales de productos y procesos de producción). 
Gráfica 4. Dimensiones de la capacidad tecnológica ${ }^{5}$

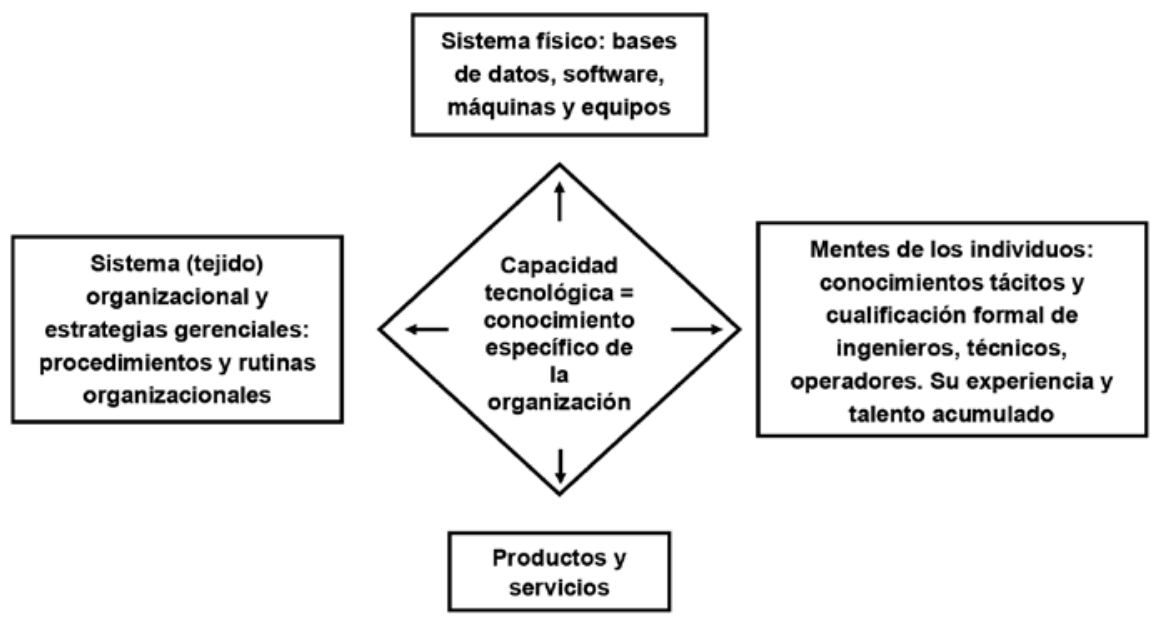

La capacidad tecnológica de una empresa se construye y se almacena en sus sistemas técnicos y físicos, en su capital humano y el conocimiento tácito, en sus sistemas de organización, de gestión e institucionales, y se evidencia en sus productos y servicios (Gráfica 4). Por tanto, existe una relación inseparable entre las cuatro dimensiones de las capacidades tecnológicas, como aspectos claves e intrínsecos al contexto de la empresa, región o país donde se desarrollan (Figueiredo, 2004).

En el caso latinoamericano, muchos estudiosos han evidenciado que ha sido posible cierto grado de aprendizaje y creación de capacidades tecnológicas en las empresas de la región, a partir de las tecnologías importadas, lo cual se ha considerado que en alguna medida contradice la teoría de la dependencia (Casas, 2004). Incluso se ha afirmado que una estrategia orientada a generar capacidades comunes, es decir, como producto de la agrupación de científicos y centros de $\mathrm{I}+\mathrm{D}$ latinoamericanos, permitirá lograr resultados que pueden equipararse a los de un país industrializado de tamaño medio (Albornoz, 2001).

En este orden de ideas, para las empresas que atienden los mercados de la base de la pirámide, como es el caso de las Pymes que caracterizan la mayoría de los aparatos productos latinoamericanos, son fundamentales los procesos de gestión y desarrollo de capacidades tecnológicas, que se traduzcan en bienes y servicios de calidad y oportunos para las necesidades de esta población, y que sean la base de procesos de innovación que contribuyan a un mayor desarrollo tecnológico local.

\section{Consideraciones sobre la valoración de tecnología en la base de la pirámide}

La valoración tecnológica ha sido definida por diversos autores; sin embargo, su concepto académico se comenzó a explorar en años recientes ( $\mathrm{Li} \&$ Chen, 2006). Con una aproximación amplia, Elói \& Santiago (2008) indican que el propósito de la valoración no es predecir el valor exacto de la tecnología, sino proporcionar un valor esperado que capture los riesgos e incertidumbres inherentes al proceso de innovación tecnológica. Adicionalmente, la valoración se orienta a la determinación del valor intrínseco de un activo tecnológico, a diferencia del pricing o fijación de su precio (LES, 2008). En general, se evidencia que en la literatura existen diversos conceptos sobre valoración tecnológica, algunos más restringidos que otros, supeditados a la conceptualización de tecnología que se maneje y al contexto en que se desarrolle el proceso de valoración.

5 Fuente: Figueiredo (2005). 
El concepto de tecnología ha cambiado a lo largo del tiempo, enriqueciéndose con aportes de diversos autores y con la tendencia a ser integrador de las diversas posturas que han ido surgiendo. Desde la visión Taylorista, la tecnología se consideraba como un medio de producción fundamentalmente tangible, si bien más adelante se empezó a reconocer el papel del conocimiento embebido en estos bienes, hablándose de paquetes tecnológicos, hasta llegar una visión extendida que también incluye las capacidades tecnológicas organizacionales. En este sentido, de acuerdo con Angelo, Domenico, Luigi \& Iacobelli (2008), puede hacerse referencia a una definición restringida y una definición amplia de la tecnología, la primera relacionada con los elementos tangibles y el conocimiento técnico asociado, mientras que el concepto amplio abarca la capacidad tecnológica total de los sistemas productivos.

Con una visión restringida de la tecnología, la conceptualización de la valoración tecnológica está asociada principalmente a la generación de innovaciones radicales, propias de contextos más avanzados. Además, los métodos utilizados, en su mayoría cuantitativos (flujo de caja descontado, método de ingresos, método de costos, opciones reales) han sido adaptados de los que se aplican para valorar otro tipo de activos, por lo cual aún existe un campo importante para la investigación en esta área, ya que sigue siendo un arte más que una ciencia (Hunt, Probert, Wong \& Phaal, 2003). Pero ante la realidad de los países latinoamericanos, en donde las capacidades de acumulación tecnológica y el aprendizaje son las opciones con mayor aplicabilidad para avanzar tecnológicamente, la tecnología y su valoración requieren ser analizadas desde una perspectiva amplia que incluya tanto los aspectos tangibles como los intangibles.

\subsection{Valoración tecnológica ampliada}

El valor de la tecnología requiere entenderse en diferentes dimensiones, no solo la económica, teniendo en cuenta la relevancia del factor tecnológico y su definición amplia, que implica considerar otros impactos además del económico. Así mismo, una visión ampliada de la valoración tecnológica puede dar mayor pertinencia a este tipo de procesos en el ámbito de la base de la pirámide, al incluir el aspecto de las capacidades y el aprendizaje tecnológico. A continuación se plantean algunas consideraciones sobre la valoración tecnológica ampliada:

La valoración de tecnología para la base de la pirámide se enriquece al retomar la conceptualización amplia de la tecnología, teniendo en cuenta la importancia de los elementos intangibles como las capacidades tecnológicas en el ámbito de los países en desarrollo, como los latinoamericanos.

La valoración de tecnología puede ser analizada como un proceso, lo cual permite pensar en términos de dinamicidad y transcurso del tiempo. La filosofía de procesos puede ser tomada como base para un análisis conceptual; esta rama de la filosofía señala que la existencia natural es mejor entendida en términos de procesos en lugar de cosas, o modos de cambio en lugar de estabilidades fijas; el cambio -ya sea físico, orgánico, sicológico, etc.- es un rasgo predominante de lo real (Rescher, 2008; Seibt, 2013). $\mathrm{Al}$ analizar la valoración tecnológica en términos de cambio, se busca abarcar características como la flexibilidad y la adaptación, puesto que esta requiere responder a las dinámicas del proceso de adquisición (o generación), asimilación y dominio de los activos tecnológicos por parte de la organización, y esto ocurre en función del tiempo. Así mismo, esta visión facilita involucrar la evolución de las organizaciones en la base de la pirámide, la gran mayoría de tamaño pequeño, para afianzarse tecnológicamente y ganar madurez en el mercado; dicha evolución se ve afectada por las condiciones específicas del contexto en el que ocurre.

Respecto a los preceptos epistemológicos subyacentes en la valoración tecnológica, caracterizados por una visión mecanicista (aspecto que se mencionó en la Introducción), una consideración adicional es buscar la complementación de la valoración tecnológica desde un enfoque organicista, de manera que se agreguen al proceso atributos como la dinámica, la complejidad y el carácter sistémico. Una propuesta que los autores de este artículo están desarrollando y espera publicarse prontamente, recurre a elementos biológicos para complementar la valoración tecnológica, tomando en cuenta que en el estudio de los organismos vivos, así como de la tecnología, no siem- 
pre es aplicable el enfoque mecanicista, ya que este tipo de sistemas se caracteriza por su complejidad. Dicha propuesta se relaciona con la consideración de la viñeta anterior, dado que el referente biológico seleccionado es la evolución y su posibilidad de extrapolación a sistemas no biológicos.

La valoración tecnológica puede estudiarse desde el punto de vista interno a la organización, como un mecanismo para determinar el valor de las tecnologías que esta posee y que no necesariamente serán comercializadas. Se tiene presente el hecho de que en la base de la pirámide se cuenta con bajo potencial de generación de tecnología, por lo cual, el concepto de valoración no siempre puede orientarse a tecnologías emergentes o que se busca comercializar. Se busca entonces que el concepto de valoración tecnológica aborde otros aspectos relacionados con la potencialidad de la innovación en países en desarrollo, y no se circunscriba a aquellas innovaciones, habitualmente de producto, que pretenden ser dispuestas en el mercado como objetivo primario de la organización que las obtuvo.

Si se intenta establecer una definición, puede señalarse que la valoración tecnológica ampliada es la valoración en la base de la pirámide, que se entiende como un proceso amplio y dinámico, no limitado a la determinación del valor o precio de una tecnología y cuya orientación no es hacia la comercialización o hacia la valoración a futuro de tecnologías en desarrollo. Se trata entonces de un proceso para la determinación del valor de un bien tecnológico y del impacto que tiene en la organización que lo posee, lo cual implica estudiar aspectos de tecnología blanda como las capacidades tecnológicas generadas para lograr el dominio de una tecnología específica por parte de la organización; este concepto toma un punto de vista interno a la organización, para analizar las tecnologías que ella posee en su proceso productivo, con el propósito de reducir la dependencia, promover procesos de innovación y hacer más eficiente el desarrollo tecnológico en la medida en que se establece el acervo tecnológico y se decide sobre su modernización (Gráfica 5).

Al revisar en detalle esta interpretación ampliada de la valoración tecnológica, se encuentran equiva- lencias con el concepto de evaluación tecnológica, como un proceso que involucra varios aspectos de la tecnología y su aprovechamiento en las organizaciones.

La evaluación tecnológica tiene en cuenta los aspectos técnicos del negocio (Elói \& Santiago, 2008), incluye las actividades de exploración de oportunidades de valor tecnológico, identificación del valor de las tecnologías y comunicación de los resultados del proceso de evaluación (Probert, Farrukh, Dissel \& Phaal, 2011), y como fundamento de la planeación y la estrategia tecnológica, debe incluir aspectos del ambiente externo así como las capacidades técnicas organizacionales (Hou, Lu \& Han, 2008). De acuerdo con estos últimos autores, la evaluación tecnológica relaciona las tecnologías disponibles en la empresa con las capacidades organizacionales de innovación, de transferencia de tecnología, de aprendizaje y dominio de la tecnología y de producción; esta relación depende de la posición competitiva que otorgue la tecnología al ser clave, emergente o "próxima estrella", pasiva o que deba ser abandonada.

Así mismo, Jolly (2012) propone que para la evaluación tecnológica se tomen en cuenta criterios como los recursos tecnológicos de la empresa respecto a su origen, su relación con el negocio central, la experiencia acumulada en campo, las patentes registradas, el valor de los laboratorios y del equipo, las competencias del equipo de trabajo para la $\mathrm{I}+\mathrm{D}$ y para la investigación aplicada, así como el desarrollo de nuevas competencias.

Por tanto, la valoración tecnológica ampliada contribuye a evidenciar las potencialidades de cada organización para la innovación así como su nivel actual y sus perspectivas, lo cual resulta clave para aquellas que atienden mercados orientados a la base de la pirámide y que se encuentran insertas en las dinámicas propias de contextos menos desarrollados. Es oportuno aclarar que tomar en cuenta criterios como las competencias organizacionales para realizar $\mathrm{I}+\mathrm{D}$ o innovar, no ocasiona que la valoración tecnológica ampliada no sea aplicable a las pymes de la base de la pirámide que, en general, no han desarrollado estas capacidades: el propósito inicial es que el análisis a través de los procesos de valoración tecnológica 
Gráfica 5. Capacidades y valoración de tecnología en la Base de la Pirámide ${ }^{6}$

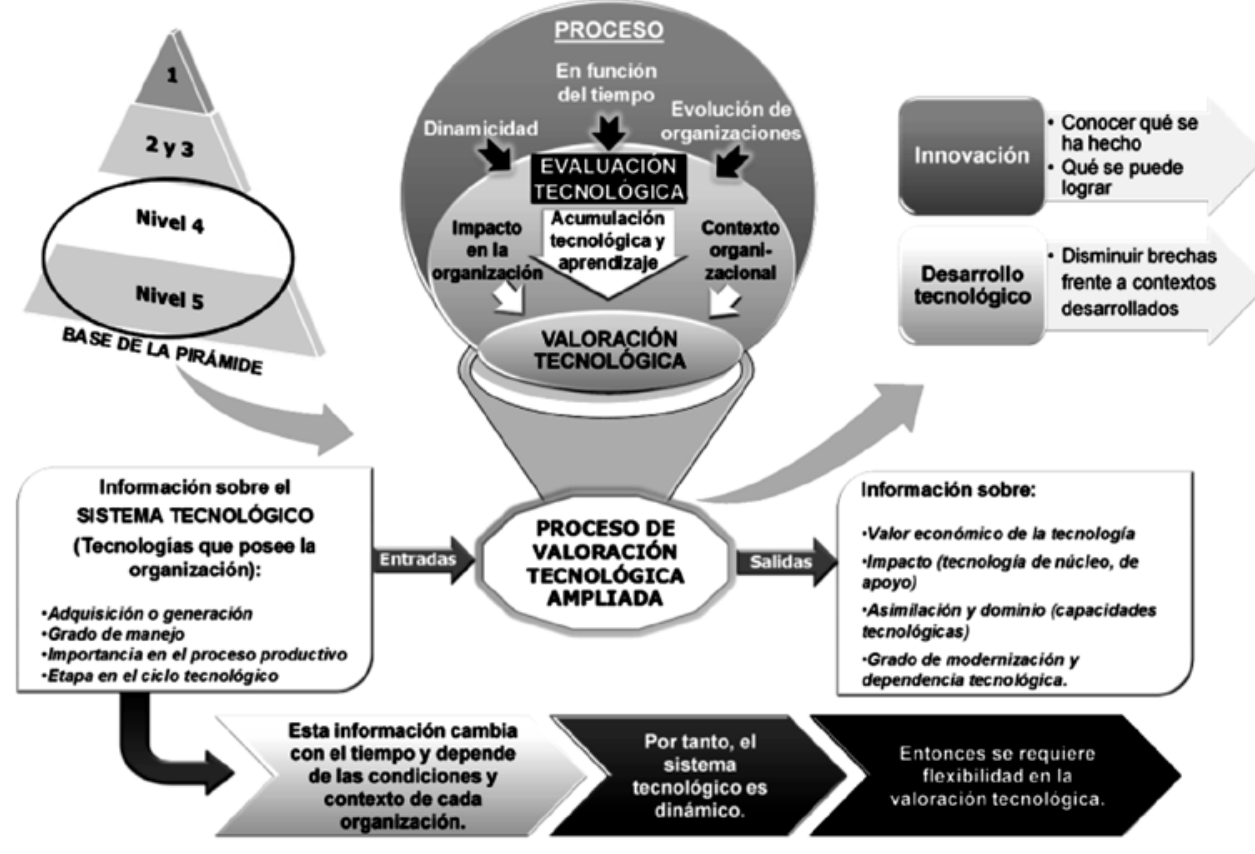

ampliada aporte a que cada empresa detecte fallas y oportunidades de modernización y de cierre de brechas tecnológicas respecto a empresas de países industrializados, aunque siempre con la premisa de lograr pertinencia frente a las necesidades locales.

\section{Conclusiones}

La innovación y el desarrollo tecnológico son aspectos prioritarios que deben fomentarse en las empresas de la base de la pirámide, en donde se ubica gran parte de la población de países como los latinoamericanos, en búsqueda de superar la dependencia tecnológica y atender oportunamente las necesidades de los mercados locales, logrando la vinculación de la población de menores recursos en sus procesos de creación de valor.

Las tecnologías características de la base de la pirámide se encuentran en las últimas fases del ciclo tecnológico, pero este rasgo no puede ser un obstáculo para el aprendizaje que lleve a procesos de innovación. Las tecnologías maduras que emplean los productores locales para satisfacer las demandas de la población de zonas menos desarrolladas, pueden articularse con tecnologías emergentes, en crecimiento o en expansión para garantizar la logística que permita llevar los productos y servicios a los lugares en que son necesitados; así mismo, debe buscarse la generación de capacidades en torno a estas tecnologías, lo cual implica sacar provecho de los recursos disponibles localmente y complementarlos con tecnologías y desarrollos de países industrializados.

En los procesos de valoración tecnológica en la base de la pirámide se debe tomar en cuenta que en este contexto aún son escasas las capacidades de generación de tecnología y de innovaciones de tipo radical, y por tanto, tales procesos serán más pertinentes si se orientan hacia otros aspectos de mayor relevancia como el aprendizaje tecnológico y la acumulación de capacidades al interior de las organizaciones, lo cual surge con base en una conceptualización am-

6 Fuente: Elaboración propia. 
plia de la tecnología que no se restringe al aspecto tangible. A partir de este hecho, en este documento se plantearon consideraciones sobre lo que se denominó valoración tecnológica ampliada, enfocada en los productores de la base de la pirámide, dadas las particularidades con las que operan y los mercados a los que atienden.

\section{Referencias}

Albornoz, M. (2001). Política científica y tecnológica: una visión desde América Latina. En: Revista Iberoamericana de Ciencia, Tecnología, Sociedad e Innovación, 1(4): 1 - 19.

Angelo, R., Domenico, C., Luigi, I. \& Iacobelli, M. (2008). Technology valuation for biotechnology: techniques for valuation of intellectual property. IBIC Conference. Italia.

Bell, M. \& Albu, M. (1999). Knowledge systems and technological dynamism in industrial clusters in developing countries. En: World development, 27 (9): 1715-1734.

Bell, M. \& Pavitt, K. (1993). Technological accumulation and industrial growth: contrasts between developed and developing countries. En: Industrial and corporate change, 2 (2): 157-210.

BID. (2011). Proceedings of BASE: The 1st International Forum for the Development of the Base of the Pyramid in Latin America and the Caribbean. Sao Paulo, Brasil, Banco Interamericano de Desarrollo.

Calderón, B. \& Silva, V. (2009). Trabajar con la base de la pirámide o negocios inclusivos: una expresión de responsabilidad social estratégica. Sexto Congreso del Comité Científico de l' ADERSE Grenoble, Francia: Groupe ESC-Pau.

Casado, F., Lariu, A., Mutis, J. \& Sánchez, P. (2009). Desarrollo de negocios en los países de bajos ingresos. Crecimiento empresarial y creación de valor social. Cátedra de Responsabilidad social corporativa. Barcelona, Universidad Pompeut Fabra.

Casas, R. (2004). Conocimiento, tecnología y desarrollo en América Latina. En: Revista Mexicana de Sociología, 66: 255-277.

CEPAL (2011). Políticas para la innovación en las pequeñas y medianas empresas en América Latina. En: Dini, M. \& Stumpo, G. (eds.) Colección documentos de proyectos. Comisión Económica para América Latina y el Caribe - CEPAL.

Doerr, K., Gates, W. \& Mutty, J. (2006). A hybrid approach to the valuation of RFID/MEMS technology applied to ordnance inventory. En: International Journal of Production Economics, 103(2): 726-741

Dutrénit, G. (2001). El papel de las rutinas en la codificación del conocimiento en las firmas. En: Análisis Económico, 16 (34): 211-230.

Eichner, T., Gemünden, H. \& Kautzsch, T. (2007). What is technology worth? En: The Journal of Investing, 16(3): 96-103.

Elói, D. \& Santiago, L. (2008). Avaliar X valorar novas tecnologias: desmistificando conceitos. Consultado el 2-5-2011 en: www. institutoinovacao.com.br/downloads/Avaliar x Valorar 1.pdf

Figueiredo, N. (2001a). Acumulação de competências tecnológicas e processos de aprendizagem; estruturas conceituais e experiências de empresas no Brasil. Rio de Janeiro, Fundação Getúlio Vargas, Escola Brasileira de Administração Pública e de Empresas.
Figueiredo, N. (2001b). Technological learning and competitive performance. Reino Unido: Edward Elgar Pub.

Figueiredo, N. (2004). Aprendizagem tecnológica e inovação industrial em economias emergentes: uma breve contribuição para o desenho e implementação de estudos empíricos e estratégias no Brasil. En: Revista Brasileira de Inovação, 3 (2): 323-361.

Figueiredo, N. (2005). Acumulação tecnológica e inovação industrial: conceitos, mensuração e evidências no Brasil. En: Sao Paulo em perspectiva, 19 (1): 54-69.

Fúquene, M. (2011). Formulación metodológica del pronóstico como herramienta para el desarrollo tecnológico (Maestría en Ingeniería Industrial Tesis de grado, Universidad Nacional de Colombia, Bogotá).

Hou, J., Lu, Q. \& Han, Y. (2008). A strategic framework for technology evaluation. International Conference on Information Management, Innovation Management and Industrial Engineering: 1-4.

Hunt, F., Probert, D., Wong, J. \& Phaal, R. (2003). Valuation of technology: exploring a practical hybrid model. PICMET: 47-53. IEEE.

Jaramillo, H., Lugones, G. \& Salazar, M. (2001). Manual de Bogotá: normalización de indicadores de innovación tecnológica en América Latina y el Caribe. Bogotá: Colciencias.

Jiménez, C. \& Castellanos, O. (2011). Hacia un enfoque multidimensional y dinámico de la valoración de tecnología. VI Congreso Internacional de la Red de Investigación y Docencia en Innovación Tecnológica RIDIT, Manizales, Colombia.

Jiménez, C. \& Castellanos, O. (2012). Technology and its value at the bottom of the pyramid. Portland International Conference on Management of Engineering and Technology: 1355-1360. Vancouver, Canadá: PICMET.

Jiménez, C. \& Castellanos, O. (2013). El valor de la tecnología: enfoques novedosos para su determinación. En: Journal of Technology Management and Innovation - JOTMI, 8 (Special edition ALTEC): 92-103.

Jiménez, C., Cristancho, A. \& Castellanos, O. (2011). Papel de las capacidades en la valoración tecnológica. En: Revista Ingeniería e Investigación, 31 (2): 112-123.

Jolly, D. (2012). Development of a two-dimensional scale for evaluating technologies in high-tech companies: An empirical examination. En: Journal of Engineering and Technology Management, 29 (2): 307-329.

Kakati, M. \& Dhar, U. (1991). Investment justification in flexible manufacturing systems. En: Engineering Costs and Production Economics, 21 (3): 203-209.

Kaplan, R. (1986). Must CIM be justified by faith alone? En: Harvard Business Review (March-April): 87-95.

Kolk, A., Rivera-Santos, M. \& Rufín, C. (2013). Reviewing a decade of research on the 'base/bottom of the pyramid' (BOP) concept. En: Business \& Society (en prensa): 1-40.

Leonard-Barton, D. (1998). Nascentes do saber: criando e sustentando as fontes de inovação. Rio de Janeiro: Fundação Getulio Vargas.

LES (2008). Technology valuation and License negotiation course. The Licensing Executives Society International.

Li, Y. \& Chen, Y. (2006). Managing technology: the technology valuation approach. PICMET: 535-540. Turquía: IEEE.

Maculan, A., Jiménez, C. \& Castellanos, O. (2014). Aprendizaje en el proceso de incubación de empresas de base tecnológica. En: Revista Tecnura (en prensa). 
Malaver, F. \& Vargas, M. (2006). Capacidades tecnológicas, innovación y competitividad de la industria de Bogotá y Cundinamarca. Bogotá: Cámara de Comercio de Bogotá, Observatorio Colombiano de Ciencia y Tecnología.

OCDE\& CEPAL. (2012). Perspectivas económicas de América Latina 2013. Políticas de Pymes para el cambio estructural. Consultado el 20-6-2013 en: http://dx.doi.org/10.1787/leo-2013-es

Pérez, C. (2001). El cambio tecnológico y las oportunidades de desarrollo como blanco móvil. En: Revista de la CEPAL, (75): 115-136.

Pérez, C. (2005). Revoluciones tecnológicas y capital financiero. La dinámica de las grandes burbujas financieras y las épocas de bonanza. México: Siglo XXI.

Pérez, C. (2009). Innovación y crecimiento. Comprender la dinámica y el cambio de las oportunidades para América Latina. En: Innovación y competitividad en la sociedad del conocimiento: 21-42. Plaza y Valdes.

Prahalad, C. (2005). La fortuna en la base de la pirámide. Barcelona: Ediciones Granica.

Prahalad, C. (2010). La nueva oportunidad de negocios en la base de la pirámide. Bogotá: Editorial Norma.

Prahalad, C. \& Hart, S. (2002). The fortune at the bottom of the pyramid. En: Strategy \& Business, (26): 2-14.

Probert, D., Farrukh, C., Dissel, M. \& Phaal, R. (2011). Towards a process framework for assessing the potential value of tech- nologies. Portland International Conference on Management of Engineering and Technology: 1-10. Portland, OR, USA: PICMET, IEEE.

Raafat, F. (2002). A comprehensive bibliography on justification of advanced manufacturing systems. En: International Journal of Production Economics, 79(3): 197-208.

Rescher, N. (2008). Process philosophy. En: Zalta, E. (ed.) The Stanford Encyclopedia of Philosophy (Fall 2008). Stanford, CA: The Metaphysics Research Lab, Stanford University.

Salom, G. \& Shulterbrandt, S. (2002). Las microempresas, las Pymes y Latinoamérica. En: Revista Inter Forum. Consultado el 12-10-2012 en: http://www.revistainterforum.com/espanol/ articulos/020302artprin3.html

Seibt, J. (2013). Process Philosophy. En: Zalta, E. (ed.) The Stanford Encyclopedia of Philosophy (Fall 2013 ed.). Stanford, CA: The Metaphysics Research Lab, Stanford University.

The Economist (2008). El futuro de la tecnología. Colección Finanzas y Negocios. Buenos Aires: Editorial Cuatro Media.

Vaccarezza, L. (2011). Ciencia, tecnología y sociedad: el estado de la cuestión en América Latina. En: Ciencia y tecnología social, 1(1): 42 - 64.

Viotti, E. (2002). National learning systems: a new approach on technological change in late industrializing economies and evidences from the cases of Brazil and South Korea. En: Technological Forecasting and Social Change, 69 (7): 653-680. 